\section{Hydroxychloroquine is neutral in risk of chronic kidney disease in patients with systemic lupus erythematosus}

With great interest, we read the lupus nephritis recommendations article by Fanouriakis et al. ${ }^{1}$ The authors highlighted that hydroxychloroquine (HCQ) use is recommended for all lupus nephritis patients to reduce risk of kidney flares, end-stage kidney disease (ESKD) and death. The authors also recommended that a reduction of 50\% HCQ dose in patients with glomerular filtration rate less than $30 \mathrm{~mL} / \mathrm{min}$.

We agree with the authors that HCQ is an important background therapy for all systemic lupus erythematosus (SLE) and lupus nephritis patients. However, the dose adjustment in patients with renal impairment should be more evidence-based. In the FDA (Food and Drug Administration) website (www.accessdata.fda. gov), information of 'range for renal clearance of unchanged drug was approximately $16 \%$ to $30 \%$ and did not correlate with creatinine clearance; therefore, a dosage adjustment is not required for patients with renal impairment' were disclosed.

Furthermore, previous studies had been debating on the effect of HCQ in chronic kidney diseases (CKD). ${ }^{2}{ }^{3}$ Pokroy-Shapira $e t$ $\mathrm{al}^{4}$ investigated 256 lupus patients for up to 25 years and found that HCQ use was negatively associated with risk of earlier CKD. We believed that evidence of HCQ in preventing ESKD and death, or even lupus nephritis flare were limited. Therefore, we designed a retrospective cohort study from population-based data set to examine the association using HCQ and their risk of subsequent CKD in patients with SLE.

In this study, we analysed Taiwan's National Health Insurance Research Database from 1997 to 2013, which provides a strongly reliable huge database and encompasses approximately $99.9 \%$ of the Taiwan population. A total of 2050 newly diagnosed SLE patients with ICD-9 (International Classification of Diseases, Ninth Revision) codes 710.0 between 2000 to 2012 were included. After excluding patients with prior CKD and HCQ never-users, a total of 783 SLE patients who had HCQ treatment that began at -90 and +365 days from diagnosis with SLE individuals were enrolled and divided into two groups according to their prescription coverage days. Group 1 had prescription of HCQ for less than 90 days, and group 2 had HCQ prescription for more than 90 days within 1 year. The baseline characteristics of both groups were comparable after 1:2 age/sex matching and 1:1 propensityscore matching on urbanisation, hospitalisation days, comorbidities and co-medications. The cumulative incidence rate of SLE was calculated for up to 14 years with Kaplan-Meier curves. The Cox proportional regression model was used to examine HR of developing subsequent CKD among two groups.

The results revealed that the cumulative incidence of $\mathrm{CKD}$ showed no significant difference between two groups (figure 1). After adjusting for age, urbanisation, length of hospital stays and possible confounders, the adjusted HR of developing CKD among the >90 days HCQ group was 1.295 (95\% CI 0.395 to 4.247), compared with $<90$ days users, indicating no statistical difference.

In conclusion, our retrospective population-based cohort study showed that HCQ use in SLE patient is neutral in subsequent risk of CKD.

Chia-Ying Wu $\odot,{ }^{1}$ Magdalene Tan, ${ }^{1}$ Jing-Yang Huang, ${ }^{2,3}$ Jeng-Yuan Chiou, ${ }^{4}$ James Cheng-Chung Wei ${ }^{3,5}$

${ }^{1}$ School of Medicine, Chung Shan Medical University, Taichung, Taiwan

'Department of Medical Research, Chung Shan Medical University Hospital, Taichung City, Taiwan

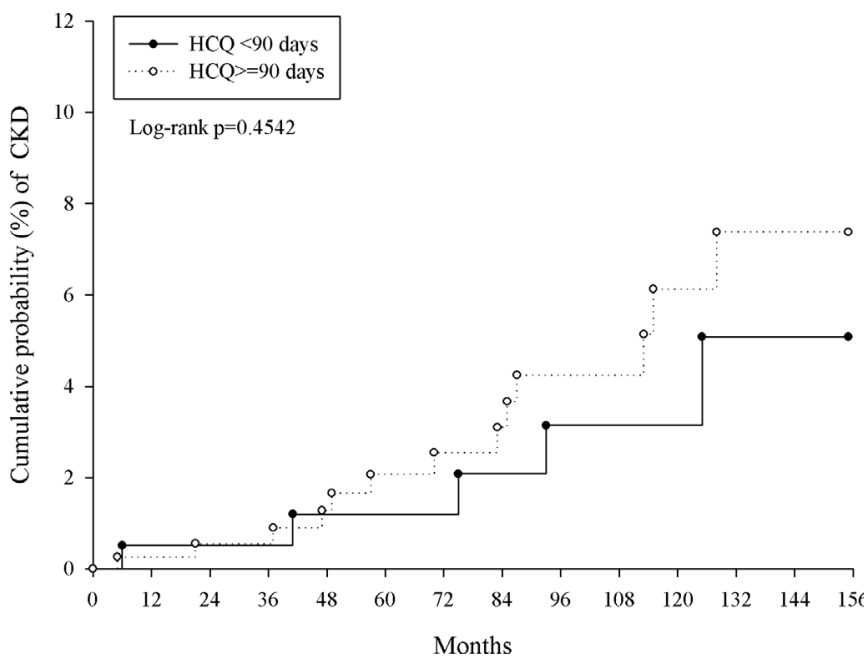

Figure 1 The comparisons of cumulative probability of CKD in systemic lupus erythematosus patients among two HCQ groups after propensity score matching. CKD, chronic kidney diseases; HCQ, hydroxychloroquine.

${ }^{3}$ Institute of Medicine, Chung Shan Medical University, Taichung, Taiwan

${ }^{4}$ School of Health Policy and Management, Chung Shan Medical University, Taichung, Taiwan

${ }^{5}$ Division of Allergy, Immunology and Rheumatology, Chung Shan Medical University Hospital, Taichung, Taiwan

Correspondence to Dr James Cheng-Chung Wei, Institute of Medicine, Chung Shan Medical University, Taichung 402, Taiwan; wei3228@gmail.com

Acknowledgements The authors thank all patients and staffs who made this study possible.

Contributors C-YW and MT: writing and revision of the manuscript, discussion of mechanism, data interpretation. C-YH: statistical analysis, data collection and interpretation. J-YC and JC-CW: study design and supervision, revision of the manuscript. All authors discussed the results and contributed to the final manuscript.

Funding None

Competing interests None declared.

Patient and public involvement Patients and/or the public were not involved in the design, or conduct, or reporting or dissemination plans of this research.

Patient consent for publication Not required.

Ethics approval This study was approved by Chung Shan Medical University Hospital.

Provenance and peer review Not commissioned; internally peer reviewed.

(c) Author(s) (or their employer(s)) 2020. No commercial re-use. See rights and permissions. Published by BMJ.

C-YW and J-YH contributed equally.

\section{Check for updates}

To cite Wu C-Y, Tan M, Huang J-Y, et al. Ann Rheum Dis Epub ahead of print: [please include Day Month Year]. doi:10.1136/annrheumdis-2020-217728

Received 23 April 2020

Accepted 27 April 2020

\section{SLinked}

- http://dx.doi.org/10.1136/annrheumdis-2020-217804

Ann Rheum Dis 2020;0:1-2. doi:10.1136/annrheumdis-2020-217728

ORCID iD

Chia-Ying Wu http://orcid.org/0000-0001-8445-7427

\section{REFERENCES}

1 Fanouriakis A, Kostopoulou M, Cheema K, et al. 2019 update of the joint European League against rheumatism and European renal Association-European dialysis and 
transplant association (EULAR/ERA-EDTA) recommendations for the management of lupus nephritis. Ann Rheum Dis 2020:annrheumdis-2020-216924.

2 Vinet E, Bernatsky S, Suissa S. Have some beneficial effects of hydroxychloroquine been overestimated? potential biases in observational studies of drug effects: Comment on the article by Pons-Estel et al. Arthritis Rheum 2009;61:1614-5. author reply 1615
3 Lee JS, Oh JS, Kim Y-G, et al. Recovery of renal function in patients with lupus nephritis and reduced renal function: the beneficial effect of hydroxychloroquine. Lupus 2020;29:52-7.

4 Pokroy-Shapira E, Gelernter I, Molad Y. Evolution of chronic kidney disease in patients with systemic lupus erythematosus over a long-period follow-up: a single-center inception cohort study. Clin Rheumatol 2014;33:649-57. 